CERN-TH/96-345

DFTT $77 / 96$

Edinburgh $96 / 29$

GEF-TH-22/96

hep-ph/9701289

\title{
DETERMINATION OF THE BJORKEN SUM AND STRONG COUPLING FROM POLARIZED STRUCTURE FUNCTIONS
}

\author{
Guido Altarelli \\ Theoretical Physics Division, CERN, CH-1211 Geneva 23, Switzerland \\ and Terza Università di Roma, Rome, Italy \\ Richard D.Ball' \\ Department of Physics and Astronomy, University of Edinburgh, \\ Mayfield Road, Edinburgh EH9 3JZ, Scotland \\ Stefano Forte \\ INFN, Sezione di Torino, \\ Via P. Giuria 1, I-10125 Torino, Italy \\ Giovanni Ridolfi \\ INFN, Sezione di Genova, \\ Via Dodecaneso 33, I-16146 Genova, Italy
}

\begin{abstract}
We present a NLO perturbative analysis of all available data on the polarized structure function $g_{1}\left(x, Q^{2}\right)$ with the aim of making a quantitative test of the validity of the Bjorken sum rule, of measuring $\alpha_{s}$, and of deriving helicity fractions. We take particular care over the small $x$ extrapolation, since it is now known that Regge behaviour is unreliable at perturbative scales. For fixed $\alpha_{s}$ we find that if all the most recent data are included $g_{A}=1.19 \pm 0.09$, confirming the Bjorken sum rule at the $8 \%$ level. We further show that the value of $\alpha_{s}$ is now reasonably well constrained by scaling violations in the structure function data, despite the fact that it cannot yet be reliably fixed by the value of the Bjorken sum: our final result is $\alpha_{s}\left(m_{Z}\right)=0.120_{-0.008}^{+0.010}$. We also confirm earlier indications of a sizeable positive gluon polarization in the nucleon.
\end{abstract}

CERN-TH/96-345

December 1996

\footnotetext{
${ }^{1}$ Royal Society University Research Fellow
} 
Much experimental and theoretical work has been devoted in recent years to polarized deep inelastic scattering [1]. Reasonably precise data on the polarized structure functions of proton [2]-[5] and deuteron [5]-[8] have been collected down to values of $x$ near and below $x=0.01$ for $Q^{2}>1 \mathrm{GeV}^{2}$. Very recently precise results on the neutron structure function from scattering on ${ }^{3} \mathrm{He}$ targets have also become available [9, 10]. The calculation of the kernels for the perturbative QCD evolution of polarized parton distributions has recently been completed to next-to-leading order (NLO) [11], thus reaching the same level of accuracy as in the unpolarized case. Experience obtained from the small $x$ behaviour of unpolarized structure functions observed at HERA [12] is now sufficient to indicate at least qualitatively the sort of behaviour we might expect for polarized structure functions at small $x$. The purpose of this paper is to take advantage of all this accumulated knowledge and experience in order to extract from the data on polarized structure functions the polarized parton densities and their moments for comparison with theoretical expectations. In particular we will show that when all the data are included it is now possible to make a reliable test of the Bjorken sum rule [13]. We will also investigate to what extent one can use the data to make an accurate determination of $\alpha_{s}$.

For an experimental verification of the Bjorken sum rule one has to extract from the data the first moment of the difference of polarized up and down quark densities at some convenient value of $Q^{2}$. Data taken at all kinematically accessible values of $x$ and $Q^{2}$, and on all available targets, contain information relevant for the reconstruction of polarized parton densities at a given $Q^{2}$ and ought therefore to be included. The complete NLO evolution kernels [11] can be used to reduce to the same $Q^{2}$ data measured at different $Q^{2}$ for each $x$. 1 Since the evolution equations [15] for partons at a given $x$ and $Q^{2}$ depend only on the values of the parton densities at larger values of $x$ and the same $Q^{2}$, the necessary correction can only be performed through a general fit to all the data, which yields a set of polarized parton densities obeying the correct evolution equations [16, 17]. However in order to perform a fit one must start with a particular ansatz for the parton densities at some reference $Q_{0}^{2}$. Clearly the results of the fit will depend to some extent on the starting ansatz one adopts, and this dependence will induce an error in the computed first moments, and in particular in the Bjorken sum. Here we will devote special attention to this issue.

Once the data are reduced to a common $Q^{2}$ for all $x$ values, an extrapolation to unmeasured values at small and large $x$ is needed in order to obtain the first moment. The extrapolation at small $x$ is especially important 18. In most of the existing analyses, including those in the experimental papers, it has been performed by assuming a simple power behaviour based on Regge theory [19]. This leads to a rather small contribution to first moments from the small $x$ region, since the expected extrapolation is at most flat. But two main considerations are now severely undermining these attempts. First of all the data at small $x$ for $g_{1}$ indicate

\footnotetext{
${ }^{1}$ In the past this has been done by assuming that the polarization asymmetries are $Q^{2}$ independent [2, 14], but this approximation is questionable given the current precision of the data.

${ }^{2}$ Note that the behaviour at small $x$ of the input ansatz for the parton densities at $Q_{0}^{2}$ is not relevant for the evolution correction, which only depends on $x$ values larger than the smallest measured one. On the contrary the integration at small $x$ that completes a given moment is very much dependent on the small $x$ behaviour of the input distributions, as we shall see.
} 
a clear departure from the nearly constant behaviour expected from Regge theory, pointing to a more singular behaviour which is moreover different for protons and neutrons. Second, we have learnt from HERA experiments that the rise of unpolarized structure functions is also much steeper than that predicted by Regge theory [12]. Although Regge theory seems to work reasonably well at scales typical of soft hadronic physics, at larger scales the effects of perturbative QCD evolution are superimposed to give a rise which gets steeper and steeper as $Q^{2}$ increases [20, 21]. A similar phenomenon is to be expected for the polarized structure functions [16, 22], so the results of a naive Regge extrapolation are not justified if one wants to consider first moments in the perturbative region: small $x$ contributions to first moments can be relatively large, especially as $Q^{2}$ increases.

Here we will discuss alternative extrapolation procedures and the errors associated with them. Our guiding principles will be the validity of Regge predictions at low $Q^{2}$, the buildup with $Q^{2}$ of the effect of the QCD evolution and the limits on the size of polarized densities imposed by the corresponding unpolarized densities. From these starting points we will estimate the uncertainty in the small $x$ extrapolation, which when combined with the evolution corrections and the more standard sources of error will allow us to quantify the extent to which the Bjorken sum rule may be tested using existing data. In practice we will do this by deriving from the data the value of $g_{A}$ and the associated error for an appropriate range of values of $\alpha_{s}$.

We will then consider the determination of $\alpha_{s}$ from the polarized deep inelastic scattering data. Previous attempts in this direction [23] have assumed the validity of the Bjorken sum rule, and used a value for the Bjorken integral obtained from the first moments given by the various experimental collaborations, and thus based on naive Regge extrapolation at small $x$. However when the effects of perturbative evolution on the small $x$ extrapolation are properly taken into account, the evaluations of the first moments must be revised: their errors then turn out to be considerably increased, and the determination of $\alpha_{s}$ from the Bjorken sum rule no longer works so well. However, we are able to show that a much better determination of $\alpha_{s}$ may be obtained if all the available data and not only the Bjorken integral are used in the analysis: the comparison of the data at small and large $Q^{2}$ in the measured range of $x$ then leads to a reasonably precise measurement of $\alpha_{s}$.

A similar procedure to that described for the Bjorken sum rule clearly also applies to the determination of other first moments, and in particular to the helicity fractions carried by the gluon density and different flavours of precisely defined quark densities. Such determinations using NLO evolution kernels have already been presented in refs. [17, 24]. What is new here, beyond the inclusion of the recent new experimental results [8]-[10], is a careful analysis of the dependence of the results on the assumed form of the input parton densities and thereby on the extrapolation to small $x$. As a result we confirm the conclusion, first reported in ref. [17], that the data indicate the presence of a sizeable positive gluon polarization in the proton, as conjectured several years previously [25]-27].

Finally, we explicitly discuss the possibility of a direct determination of $\alpha_{s}$ through the Bjorken sum rule from the measurement of the isotriplet first moment of $g_{1}$. If it were possible to determine this first moment very accurately, one might then be able to take advantage 23. 
of the fact that radiative corrections to the nonsinglet first moment are know to NNNLO, that is to order $\alpha_{s}^{3}$. We show that, with current data, even though the central value of $\alpha_{s}$ obtained with this procedure is quite compatible with that of the NLO analysis the experimental error is considerably larger, due to the uncertainty in the contribution to the isotriplet first moment from the unmeasured small $x$ region.

\section{Polarized Structure Functions and Partons}

We begin by summarising various results on the relation between structure functions and polarized parton distributions, and their behaviour at small $x$, which will be important for the following discussion.

\subsection{Defining Polarized Parton Densities}

The structure function $g_{1}$ is related to the polarized quark and gluon distributions by [17]

$$
g_{1}\left(x, Q^{2}\right)=\frac{\left\langle e^{2}\right\rangle}{2}\left[C_{N S} \otimes \Delta q_{N S}+C_{S} \otimes \Delta \Sigma+2 n_{f} C_{g} \otimes \Delta g\right],
$$

where $\left\langle e^{2}\right\rangle=n_{f}^{-1} \sum_{i=1}^{n} e_{i}^{2}, \otimes$ denotes convolution with respect to $x$, and the nonsinglet and singlet quark distributions are defined as

$$
\Delta q_{N S} \equiv \sum_{i=1}^{n_{f}}\left(\frac{e_{i}^{2}}{\left\langle e^{2}\right\rangle}-1\right)\left(\Delta q_{i}+\Delta \bar{q}_{i}\right), \quad \Delta \Sigma \equiv \sum_{i=1}^{n_{f}}\left(\Delta q_{i}+\Delta \bar{q}_{i}\right),
$$

where $\Delta q_{i}$ and $\Delta \bar{q}_{i}$ are the quark and antiquark distributions of flavor $i$ and $\Delta g$ is the polarized gluon distribution. The evolution equations for the polarized parton densities are given by

$$
\begin{aligned}
\frac{d}{d t} \Delta q_{N S} & =\frac{\alpha_{s}(t)}{2 \pi} P_{q q}^{N S} \otimes \Delta q_{N S} \\
\frac{d}{d t}\left(\begin{array}{c}
\Delta \Sigma \\
\Delta g
\end{array}\right) & =\frac{\alpha_{s}(t)}{2 \pi}\left(\begin{array}{cc}
P_{q q}^{S} & 2 n_{f} P_{q g}^{S} \\
P_{g q}^{S} & P_{g g}^{S}
\end{array}\right) \otimes\left(\begin{array}{c}
\Delta \Sigma \\
\Delta g
\end{array}\right),
\end{aligned}
$$

where $t=\log Q^{2} / \Lambda^{2}$. The coefficient functions $C$ and the polarized splitting functions $P$ are now known at LO [15] and NLO [11]. Moments of coefficient functions and parton densities are defined as $f(N)=\int_{0}^{1} d x x^{N-1} f(x)$ and denoted by $C\left(N, \alpha_{s}\right), \Delta q_{N S}\left(N, Q^{2}\right), \Delta \Sigma\left(N, Q^{2}\right)$ and $\Delta g\left(N, Q^{2}\right)$.

As is well known [1], the definition of the singlet quark density $\Delta \Sigma\left(x, Q^{2}\right)$ must be carefully specified, since its first moment is maximally scheme dependent. This follows from the fact that, due to the axial anomaly, $\alpha_{s}(t) \Delta g\left(1, Q^{2}\right)$ is scale independent at LO. This implies that even asymptotically the ambiguity in $\Delta \Sigma\left(1, Q^{2}\right)$ is necessarily of the same order as its size. For a sensible comparison with the constituent quark spin one must thus define $\Delta \Sigma\left(1, Q^{2}\right)$ in such 
a way that it is scale independent [25, 28]: $\Delta \Sigma\left(1, Q^{2}\right)=\Delta \Sigma(1)$. This is the definition we will adopt here. We then have

$$
\Gamma_{1}\left(Q^{2}\right) \equiv \int_{0}^{1} d x g_{1}\left(x, Q^{2}\right)=\frac{\left\langle e^{2}\right\rangle}{2}\left[C_{N S}\left(1, \alpha_{s}(t)\right) \Delta q_{N S}(1)+C_{S}\left(1, \alpha_{s}(t)\right) a_{0}\left(Q^{2}\right)\right]
$$

with $a_{0}$ the singlet axial charge:

$$
a_{0}\left(Q^{2}\right)=\Delta \Sigma\left(1, Q^{2}\right)-n_{f} \frac{\alpha_{s}(t)}{2 \pi} \Delta g\left(1, Q^{2}\right) .
$$

The higher moments of the singlet quark distribution are also scheme dependent, although in a less dramatic way. Various schemes were discussed in ref. [17, 29] and the dependence of the results of the analysis on the choice of scheme was studied. Here we do not come back to this issue but instead simply adopt the AB scheme as defined in ref. [17].

\subsection{Small $x$ Behaviour}

In view of the need to extrapolate the data to $x=0$ in order to compute moments, it is important to summarise the current understanding of the small $x$ behaviour of structure functions. For the unpolarized singlet quark and gluon distributions the QCD evolution equations (3) lead to the following asymptotic behaviour at small $x$ [20, 21]:

$$
\begin{aligned}
x g & \sim \sigma^{-1 / 2} e^{2 \gamma \sigma-\delta \zeta}\left(1+\sum_{i=1}^{n} \epsilon^{i} \rho^{i+1} \alpha_{s}^{i}\right), \\
x \Sigma & \sim \rho^{-1} \sigma^{-1 / 2} e^{2 \gamma \sigma-\delta \zeta}\left(1+\sum_{i=1}^{n} \epsilon_{f}^{i} \rho^{i+1} \alpha_{s}^{i}\right),
\end{aligned}
$$

where $\xi=\log x_{0} / x, \zeta=\log \left(\alpha_{s}\left(Q_{0}^{2}\right) / \alpha_{s}\left(Q^{2}\right)\right), \sigma=\sqrt{\xi \zeta}, \rho=\sqrt{\xi / \zeta}$, and the $\epsilon$ terms indicate corrections from the $n$-th perturbative order (with $n=1$ corresponding to NLO). It follows that the structure functions $x F_{1}$ and $F_{2}$ rise at small $x$ more and more steeply as $Q^{2}$ increases, though, for all finite $n$, never as steeply as a power of $x$. For all other parton distributions $f$ $\left(f=q_{N S}, \Delta q_{N S}, \Delta \Sigma, \Delta g\right)$ one has similarly [22, 16]

$$
f \sim \sigma^{-1 / 2} e^{2 \gamma_{f} \sigma-\delta_{f} \zeta}\left(1+\sum_{i=1}^{n} \epsilon_{f}^{i} \rho^{2 i+1} \alpha_{s}^{i}\right)
$$

Thus these distributions are less singular by a factor of $x$ than the singlet unpolarized distributions eq. (6), while the higher order corrections are more important at small $x$ since the exponent $i+1$ is replaced by $2 i+1$; this is because the leading small $N$ contributions to the anomalous dimensions at order $\alpha_{s}^{i+1}$ are $\left(\alpha_{s} /(N-1)\right)^{i}$ in the unpolarized singlet case, but $N\left(\alpha_{s} / N^{2}\right)^{i}$ for the nonsinglet and polarized distributions.

The limiting behaviour (6, (7) implied by the evolution equations (3) at finite order in perturbation theory would be spoiled if the series of higher order powers of $\log 1 / x$ were summed to all orders to give a powerlike behaviour in $x$, which would then overwhelm the leading terms. As is well known [30], in the unpolarized singlet channel one may obtain a result as singular as $x^{-\lambda}$, with $\lambda \sim 1 / 2$, for $x \Sigma$ and $x g$ by summation of higher order singularities in the Regge limit of $x \rightarrow 0$ at fixed $Q^{2}$ (hence fixed $\alpha_{s}$ ). However the meaning and the value of a fixed 
$\alpha_{s}$ are quite ambiguous, and it is not at all necessary a priori that such a singular behaviour is of relevance in the measured HERA region. In fact the experimental results from HERA show no evidence at all for this behaviour [21, 12]. In principle the higher order terms could be more important for the nonsinglet and polarized distributions due to the $2 i+1$ exponent in eq. (17) instead of $i+1$ in eq. (6). Indeed summing these 'double' logarithmic singularities [31, 32 appears to lead to a singular behaviour $f \sim x^{-\lambda}$ with $\lambda \sim 0.5$ for $q_{N S}$ and $\Delta q_{N S}$, and $\lambda \gtrsim 1$ for the singlet densities (suggesting that the first moment of the singlet part of $g_{1}$ could be arbitrarily large). If one were to take these theoretical predictions seriously, the errors in the small $x$ extrapolations considered below, particularly in the singlet channel, would have to be considered only as lower bounds. However the summation of 'double' logarithms is even less well founded theoretically than the summation of 'single' logarithms in the unpolarized singlet channel, and we believe that at present none of these results should be interpreted too literally [12].

Another important difference between the small $x$ behaviour of unpolarized and polarized singlet distributions is that in the unpolarized case only the gluon anomalous dimension carries the leading singularity, and consequently the rise in the singlet quark distribution is driven directly by that of the gluon, while in the polarized case all the entries in the matrix of singlet anomalous dimensions are singular, and the polarized singlet quark and gluon distributions mix. It turns out that the leading eigenvector of small $x$ evolution is then such that the singlet quark and gluon distributions have opposite sign, which means in practice that the singlet component of $g_{1}$ is driven negative at small $x$ and large $Q^{2}$ [16]. Contributions to first moments of $g_{1}$ from the small- $x$ tail thus tend to become negative when $Q^{2}$ is sufficiently large.

The purely perturbative asymptotic predictions eqs. (6,7) only hold when the input distribution at the starting scale $Q_{0}^{2}$ is relatively nonsingular: if the singularity in the input is stronger than that generated perturbatively then the input will be essentially preserved by the perturbative evolution. The rise at small $x$ will then be largely independent of $Q^{2}$, rather than becoming steeper as $Q^{2}$ increases. If we take the starting scale in the crossover region between perturbative and nonperturbative dynamics, we can presumably take the small $x$ behaviour of the input from Regge theory. For unpolarized distributions the input to the singlet distributions (given by the pomeron trajectory) is then relatively flat, and indeed the dominance of the perturbative behaviour (6) is confirmed by $F_{2}$ data from HERA [12, 21], while the input to the nonsinglet (given by the $\rho-\omega$ Reggeon trajectory) is singular, behaving as $x^{-1 / 2}$, so it is preserved by the evolution and is consistent with data from NMC and CCFR. For polarized distributions Regge theory suggests that the form of the input should be given by the $A_{1}$ trajectory, and thus flat or even vanishing, behaving as $x^{0}-x^{0.5}$ [19].

In the following we will tentatively adopt a physical picture, inspired by the HERA results, in which we assume the validity of Regge behaviour at small $x$ in the soft region (i.e. that at some input scale $Q_{0}^{2} \lesssim 1 \mathrm{GeV}^{2}$ the polarized densities are flat or vanishing) while at larger $Q^{2}$ the effect of NLO perturbative evolution is superimposed (giving a perturbative growth of the form eq. (]$\left.^{1}\right)$ ). We will also allow steeper inputs in the nonsinglet sector, provided they are consistent with the partonic constraint $\left|\Delta q_{N S}\left(x, Q^{2}\right)\right| \lesssim q_{N S}\left(x, Q^{2}\right)$ : this limits the effective small $x$ behaviour of $\Delta q_{N S}$ to a singular growth $\sim x^{-0.5}$. This picture turns out to be consistent 
with the data, and gives a constraint on the allowed growth of $\left|\Delta q_{N S}\left(x, Q^{2}\right)\right|$ in the unmeasured region which in turn limits the possible ambiguity on the Bjorken sum rule from the small $x$ extrapolation. Unfortunately in the polarized singlet sector the corresponding partonic bound is much less restrictive and one has here to rely entirely on the validity of the NLO approximation and Regge behaviour at a low enough scale.

\section{Polarized Parton Densities from $g_{1}$ Data}

We now consider in detail the problem of extracting relevant physical quantities from the existing data. We devote particular attention to the study of the dependence of the results on the assumed functional form of the input parton distributions. For this purpose, we consider a variety of possible parameterizations of the input, we evolve these up to the values of $x$ and $Q^{2}$ where data are available by solution of the evolution eqs. (3) at NLO, and we determine the free parameters of the input by a best fit of $g_{1}\left(x, Q^{2}\right)$ eq. (1) to all the data of refs. [3]- [10] with $Q^{2} \geq 1 \mathrm{GeV}^{2}$. Experimental data for $g_{1}$ are obtained from the experimentally measured asymmetries $A_{1}$ using a parameterization of the measured unpolarized structure functions $F_{2}$ [33] and $R$ [34] consistently neglecting all higher twist corrections, and, for deuterium and helium targets, accounting for effects due to the nuclear wavefunction (but not Fermi motion or shadowing) by a simple multiplicative correction [35, 9]. Throughout this section we take $\alpha_{s}\left(m_{Z}\right)=0.118 \pm 0.005$ [36] and $a_{8}=0.579 \pm 0.025$ [37] where $a_{8}$ is the $\mathrm{SU}(3)$ octet axial charge (in the proton, below charm threshold, $\Delta q_{N S}(1) \equiv \eta_{N S}=\frac{3}{4} g_{A}+\frac{1}{4} a_{8}$ ).

To begin with we parameterize the initial parton distributions at $Q_{0}^{2}=1 \mathrm{GeV}^{2}$ according to the conventional form

$$
\Delta f\left(x, Q_{0}^{2}\right)=\mathcal{N}_{f} \eta_{f} x^{\alpha_{f}}(1-x)^{\beta_{f}}\left(1+\gamma_{f} x^{\delta_{f}}\right)
$$

where $\Delta f$ denotes $\Delta q_{N S}, \Delta \Sigma$ or $\Delta g$ and $\mathcal{N}_{f}$ is a normalisation factor chosen such that the first moment of $\Delta f$ is equal to $\eta_{f}$. The signs of all parameters are left free, including the overall factors $\eta_{f}$ (although the data always choose $\eta_{f}$ to be positive). In table 1 we report the results from a fit of this class, denoted as fit A, which corresponds to:

$$
\delta_{\Sigma}=\delta_{g}=1, \quad \delta_{N S}=0.75 \quad(\text { fixed }), \quad \gamma_{\Sigma}=\gamma_{g} \quad(\text { fit A })
$$

It is particularly important to see to what extent the data fix the small $x$ behaviour i.e. the exponents $\alpha_{f}$ in eq. (8). Note that the $g_{1}$ data on protons and neutrons show a strong rise or fall at small $x$, while the deuteron data are much flatter. Thus the fitted nonsinglet quark densities at small $x$ tend to show a sharp rise while the singlet quarks tend to remain fairly flat. Starting from a generic input set of densities of the class eq. (8) we can easily end up with $\Delta q_{N S}$ considerably more singular than $\sim x^{-0.5}$, which we cannot accept since then the nonsinglet polarized distribution would eventually become larger than the unpolarized

\footnotetext{
${ }^{3}$ Although for the E154 data 10] the preliminary published values of $g_{1}^{n}$ are used directly since the experimental asymmetries are not yet available.
} 
distribution. However a more careful analysis reveals that there is a strong correlation between $\alpha_{f}$ and $\delta_{f}$. In the measured region one can easily push $\alpha_{N S}$ closer to zero by decreasing $\delta_{N S}$ from unity without appreciable changes in the quality of the fit. For example for $\delta_{N S}=1,0.75,0.5$ we find $\alpha_{N S} \sim-0.8,-0.5,-0.3$, respectively, with $\chi^{2} \sim 89,89,90$. Thus we find that the existing data do not much constrain the behaviour of the nonsinglet at asymptotically small values of $x$ : even within the simple functional form eq. (8) one still has a considerable flexibility in the asymptotic behaviour as $x \rightarrow 0$. Still, it appears that, within this class of fits, the resulting nonsinglet quark density is rather more singular at $Q_{0}^{2}=1 \mathrm{GeV}^{2}$ than we would expect from naive Regge theory, suggesting that Regge behaviour can only hold at significantly lower scales. The choice of parameters eq. (8) corresponds to a typical case which saturates the partonic constraint discussed at the end of the previous section.

In order to discuss less singular inputs, we completely change the functional form of the input densities (while keeping the initial scale at $Q_{0}^{2}=1 \mathrm{GeV}^{2}$ ). We thus choose an input in which the rise at small $x$ is at most logarithmic (fit $\mathrm{B})$ :

$$
\begin{aligned}
\Delta \Sigma & =\mathcal{N}_{\Sigma} \eta_{\Sigma} x^{\alpha_{\Sigma}}(\log 1 / x)^{\beta_{\Sigma}} \\
\Delta q_{N S} & =\mathcal{N}_{N S} \eta_{N S}\left[(\log 1 / x)^{\alpha_{N S}}+\gamma_{N S} x(\log 1 / x)^{\beta_{N S}}\right] \quad \text { (fit B) } \\
\Delta g & =\mathcal{N}_{g} \eta_{g}\left[(\log 1 / x)^{\alpha_{g}}+\gamma_{g} x(\log 1 / x)^{\beta_{g}}\right]
\end{aligned}
$$

Note that $\log 1 / x \sim(1-x)$ near $x=1$, so that the $\gamma$ terms take care of the behaviour as $x \rightarrow 1$. The results we obtain from this fit are again reported in table 1 . It is remarkable that we obtain a fit that is equally good in the measured region (as seen from the $\chi^{2}$ value) but with a quite different extrapolation in the unmeasured region. As a consequence, for example, the fitted value of $g_{A}$ changes from $g_{A}=1.17 \pm 0.05$ in fit A to $g_{A}=1.23 \pm 0.07$ in fit B. The small $x$ behavior of this fit is weaker than any power, and thus in particular compatible with the Regge prediction.

Although the logs are reminiscent of QCD evolution the functional form of fit B might perhaps appear a little ad hoc. It is thus interesting to try to generate the logarithms in a more physical way, by perturbative evolution. In this spirit we consider another set of trials, where we start the QCD evolution at a very small scale, $Q_{0}^{2}=0.3 \mathrm{GeV}^{2}$, and fit a function of the form eq. (8). The choice of such a low scale is simply used as a trick to generate an effective set of distributions at the value of $Q^{2}$ at which we begin to fit the data, i.e. $Q^{2}=1 \mathrm{GeV}^{2}$ (data with lower $Q^{2}$ being still discarded), with the logs piled up in a way entirely consistent with perturbative evolution. In table 1 we report the results from a fit with

$$
\gamma_{\Sigma}=\gamma_{g}=\gamma_{N S}=0, \quad \beta_{g}=15 \quad(\text { fixed }) \quad(\text { fit } \mathrm{C})
$$

In this class of fits, the large- $x$ behaviour of the gluon distribution can hardly be determined by the fitting procedure; therefore, we fixed $\beta_{g}=15$ at $Q_{0}^{2}=0.3 \mathrm{GeV}^{2}$, because we checked that this choice approximately corresponds to a $(1-x)^{4}$ behaviour of $\Delta g$ at $Q^{2}$ around $1 \mathrm{GeV}^{2}$. Once more the quality of the fit in the measured region is unchanged. Comparing with the results of fit $\mathrm{A}$, we see that by lowering the initial $Q_{0}^{2}$ scale all the exponents in the $x^{\alpha}$ terms have become positive in qualitative agreement with the idea that naive Regge behaviour is 
restored at a sufficiently low scale. Indeed a fit of comparable quality is obtained if we fix all exponents $\alpha_{f}$ at $Q_{0}^{2}=0.3 \mathrm{GeV}^{2}$ to the limiting value admitted by Regge theory, i.e. one half (fit D):

$$
\alpha_{f}=0.5, \quad \gamma_{\Sigma}=0, \quad \delta_{g}=1, \quad \delta_{N S}=1, \quad \text { (fit D). }
$$

The results of this fit are also shown in table 1 . The $\chi^{2}$ is now slightly worse, but the physical results do not change much, especially in the nonsinglet sector (for example the central value of $g_{A}$ is about the same in fits $\mathrm{C}$ and $\mathrm{D}$ ). One could presumably optimize the choice of the initial scale $Q_{0}^{2}$ to make the agreement with Regge theory even better.

In figs. 1a-c we display the best-fit $g_{1}$ (fit B) for protons, neutrons and deuterons at the $Q^{2}$ of the data. In figs. 2a-c we then compare the best-fit forms of $g_{1}$ corresponding to fits A-D at $Q^{2}=10 \mathrm{GeV}^{2}$. The figures show that while the four fits are reasonably close together in the measured region $(x \sim 0.003-0.03$ up to $x \sim 0.8)$, they become very different in the small $x$ region. Note that at this $Q^{2}$ value $g_{1}$ has indeed become negative at small $x$ in all cases. In fig. 3a-c we display the resulting polarized parton densities obtained from the fits at the same value of $Q^{2}$. The behaviour at small $x$ is quite different in each case. The fits $\mathrm{C}$ and $\mathrm{D}$ develop a particularly robust tail at small $x$ in the singlet sector. It is the large positively polarized gluon that drives $g_{1}$ negative at small $x$.

In table 2 we report the values obtained by computing the first moments of $g_{1}$ by integration of the four fits, both in the measured range of $x$ and in the whole range at the 'average' values of $Q^{2}$ quoted by each experiment on protons, deuterons and neutrons. We see that while the truncated moments are remarkably close to each other the complete moments show a much wider spread. We also report the values of the truncated moments obtained by evolving the data to a common scale by means of the traditional (but unjustified) assumption that the asymmetries are scale independent and then summing over the bins, and those given by the experimental collaborations with their associated total errors. The latter two values should in principle coincide and only differ because of details in the way $g_{1}$ is determined from the measured asymmetries (such as the use of different parameterizations of the unpolarized structure function $F_{2}$, or the inclusion of some higher twist corrections, as done by some experimental collaborations). The effect of the $Q^{2}$ dependence in the measured region is sizeable but smaller than the experimental error. Much larger is the indirect effect of scaling violations on the extrapolation at small $x$ because of the larger scale dependence at small $x$.

\section{Phenomenological Implications}

We will now discuss the quantitative consequences that can be derived from the results of the previous section. In particular we will discuss the status of the Bjorken sum rule, the polarization of different quark flavors and of gluons in the proton, and the determination of $\alpha_{s}$. 


\subsection{Testing the Bjorken Sum Rule}

One general way to test the Bjorken sum rule is to determine $g_{A}$ and the associated error from fitting the whole set of available data points. The error has many components to it: the error from the individual fit (based on a given functional form) due to the experimental errors on each data point, the ambiguity on the input functional form for the parton densities (which thus includes the error from the small $x$ extrapolation), the error due to the uncertainty in the values of $\alpha_{s}$ and $a_{8}$ (we take $\alpha_{s}\left(m_{Z}\right)=0.118 \pm 0.005$ [36], and $a_{8}=0.579 \pm 0.025$ [37]|尸], the error due to threshold uncertainties (estimated by varying the position of the thresholds around $m_{q}$ by a factor 2), and finally the error related to unknown higher perturbative orders (estimated by varying the renormalization and factorization scales $\mu_{R}^{2}, \mu_{F}^{2}$ by a factor of two in either direction). The error due to higher twist terms is potentially significant, because the data at low $Q^{2}$ (and thus at low $x$ ) are essential for the computation of the first moments, but difficult to estimate reliably: here we estimate it (see sect. 3.4 below) from the shift in the value of $g_{A}$ extracted from the Bjorken sum rule due to the inclusion of a higher twist correction to it according to QCD sum rule and renormalon estimates [39].

Based on the fits shown in table 1, and similar ones used to estimate theoretical errors, we find

$$
g_{A}=1.19 \pm 0.05(\exp ) \pm 0.07(\mathrm{th})=1.19 \pm 0.09,
$$

where the central value is obtained as the average between the maximum and minimum values of table 1 , and the various contributions to the theoretical error are listed in table 3 . The fitted value is to be compared with the direct measurement $g_{A}=1.257 \pm 0.003$ 40 from $\beta$-decay. Thus we find that the Bjorken sum rule is confirmed to within one standard deviation but still with an accuracy of only about $8 \%$. For the chosen value of $\alpha_{s}$ the fitted value of $g_{A}$ is below the experimental value; it would be increased by using a larger value of $\alpha_{s}$, or perhaps by the inclusion of additional corrections beyond NLO.

\subsection{Singlet First Moments}

Similarly in the singlet sector one obtains from the data values for $\eta_{q}=\Delta \Sigma(1)$ (the conserved polarized singlet quark density), for $\eta_{g}=\Delta g\left(1,1 \mathrm{GeV}^{2}\right)$ (the first moment of the polarized gluon density evaluated at $\left.Q^{2}=1 \mathrm{GeV}^{2}\right)$, and for $a_{0}\left(10 \mathrm{GeV}^{2}\right)$ (the non conserved singlet axial charge defined implicitly from the singlet part of $g_{1}$ by eq. (四)). This latter quantity approaches a finite limit at infinite $Q^{2}$ because the corresponding anomalous dimension starts at two loops, and within the present accuracy $a_{0}\left(10 \mathrm{GeV}^{2}\right)$ is equivalent to $a_{0}(\infty)$. The values for these three quantities as obtained from our representative fits are reported in table 1 . We then studied in detail, following ref. [17], the theoretical errors from the various different sources: results for these are listed in table 3. The error due to higher twists is not included since it cannot be

\footnotetext{
${ }^{4}$ The sensitivity to $a_{8}$ is in practice very weak: we have checked that variations of up to $30 \%$ have little effect on our results. Such deviations from the value of ref. [37] could be caused by SU(3) violations in hyperon semileptonic decays 38.
} 
reliably estimated but, on the basis of the corresponding error on $g_{A}$, it is expected to scarcely affect the total errors which in the singlet sector are rather large (for reasons discussed in detail in refs. [16, 17]). In comparison to ref. [17] we have a more reliable determination of the error related to the fitting procedure, and a somewhat stronger sensitivity to higher order corrections. We then find

$$
\begin{aligned}
\Delta \Sigma(1) & =0.45 \pm 0.04(\exp ) \pm 0.08(\text { th })=0.45 \pm 0.09 \\
\Delta g\left(1,1 \mathrm{GeV}^{2}\right) & =1.6 \pm 0.4(\exp ) \pm 0.8(\text { th })=1.6 \pm 0.9 \\
a_{0}(\infty) & =0.10 \pm 0.05(\exp )_{-0.10}^{+0.17}(\text { th })=0.10_{-0.11}^{+0.17}
\end{aligned}
$$

in excellent agreement with the corresponding results of ref. [17]. The physical implications are thus the same. The parameter $a_{0}(\infty)$ measures the degree of 'spin crisis': the singlet axial charge of the nucleon is still compatible with zero as it was at the beginning of the story [2]. Note that with the naive Regge extrapolation at small $x$ the experimental result for the axial singlet charge would be significantly larger, with a much smaller error: for example in ref. 41] a value $a_{0}(\infty)=0.33 \pm 0.04$ was quoted. There is also some evidence (around two standard deviations) for a positive gluon polarization in the nucleon (increasing with $Q^{2}$ as $\left.1 / \alpha_{s}\left(Q^{2}\right)\right)$. The amount of gluon polarization is large enough to allow the first moment $\Delta \Sigma(1)$ of the conserved singlet quark density to be within one standard deviation of $a_{8} \sim 0.58$, which in the absence of all $\mathrm{SU}(3)$ and chiral symmetry breaking effects, could be identified with the constituent spin fraction [42]. This can be seen as a direct confirmation of the physical explanation of the 'spin crisis', advocated in refs. [25]-27], as due to the anomaly and well described in terms of the QCD parton model (for more general possibilities, see refs. [43]).

\subsection{Determination of $\alpha_{s}$}

The above discussion on $g_{A}$ makes it clear that the determination of $\alpha_{s}$ from the Bjorken integral is adversely affected by the increased ambiguity from the small $x$ extrapolation that follows from the demise of the naive Regge behaviour at small $x$. Fortunately we find that $\alpha_{s}$ can be determined directly from the available data without extrapolation in the small $x$ region if the totality of the data is taken into account and not just the Bjorken integral. The value of $\alpha_{s}$ is then determined by the strong scaling violations needed to accommodate the difference between the data at small $Q^{2}$ from the SLAC experiments and those at larger $Q^{2}$ from the $\mathrm{SMC}$ in the common range of $x$. While $\Delta g\left(1,1 \mathrm{GeV}^{2}\right)$ is mainly fixed by the proton data, $\alpha_{s}$ is determined by the difference between proton and neutron. To show this we repeated the fits A-D but fixing $g_{A}$ to its experimental value and instead fitting $\alpha_{s}$ (in this case, fit A was performed with $\beta_{g}$ fixed at the value $\beta_{g}=4$ ). In all cases the central value was found to be close to $\alpha_{s}\left(m_{Z}\right)=0.120$. Since the different fits differ considerably in the unmeasured region, this shows that it is the behaviour in the measured region that matters. In addition, the Bjorken integral is appreciably different in the different fits A-D when $\alpha_{s}$ is kept fixed (and the value of $\Delta g\left(1,1 \mathrm{GeV}^{2}\right)$ even more so) but this difference does not affect the fitted $\alpha_{s}$ very much. Furthermore, the resolution of the discrepancy between the fitted value of $g_{A}$ eq. (13) and its experimental value would require a much larger increase of $\alpha_{s}$ if only the Bjorken integral were 
relevant for fixing $\alpha_{s}$. These results show that $\alpha_{s}$ is much better constrained by the overall pattern of scaling violations than by the Bjorken integral alone.

The theoretical uncertainties that affect this determination of $\alpha_{s}$ are listed in table 3 . The main source of uncertainty originates from higher order and higher twist contributions. Higher order corrections are estimated by varying the renormalization and factorization scales $\mu_{R}^{2}, \mu_{F}^{2}$ around $Q^{2}$. The values of table 3 are obtained varying $\mu_{R}^{2}$ and $\mu_{F}^{2}$ between $Q^{2} / 2$ and $4 Q^{2}$. A wider scale variation at the lower edge is made impossible, without leaving the perturbative domain, by the presence of data with values of $Q^{2}$ close to $1 \mathrm{GeV}^{2}$. The higher twist error is again determined from the corresponding shift in the value of $\alpha_{s}$ extracted from the Bjorken sum rule (sect. 3.4 below). We can cross-check this estimate of higher twist terms by repeating the fit while excluding all data with $Q_{0}^{2}<2 \mathrm{GeV}^{2}$. We find that the value of $\alpha_{s}$ does not change, thus suggesting that higher twist effects are indeed quite small.

We thus obtain finally

$$
\alpha_{s}\left(m_{Z}\right)=0.120_{-0.005}^{+0.004}(\exp )_{-0.006}^{+0.009}(\mathrm{th})=0.120_{-0.008}^{+0.010}
$$

This reasonably good determination of $\alpha_{s}\left(m_{Z}\right)$ could still be improved with better data: it is important to notice that without the very recent neutron data 10 the experimental error would be twice as large. We see no reason why it could not be as good as the determination from unpolarized data if more data were added and the experimental errors consequently reduced. However the theoretical error is already the dominant one; it could also be reduced by more data because it is made particularly large by the small $Q^{2}$ values of the neutron measurements.

\subsection{Determining $\alpha_{s}$ from the Bjorken sum rule}

Even though the full set of anomalous dimensions and coefficient functions are known at NLO, for the nonsinglet first moment the anomalous dimension vanishes and the coefficient function is known to order $\alpha_{s}^{3}$, i.e. at NNNLO 44. One would like to take advantage of this situation by directly determining $\alpha_{s}$ from the Bjorken sum rule [23]. This however requires first a determination of the Bjorken integral from the data. Since the latter can only be accomplished at NLO the procedure is only advantageous to the extent that this extraction can be done in a way that minimizes the necessary theoretical input.

This can be done following a procedure close to that used in the experimental papers: first one computes directly from the data the contribution to the first moment from the experimentally accessible $x$ range (applying a NLO correction to account for evolution of the data at a common scale for each experiment), then one adds to it an extrapolation over the unobserved $x$-range. The resulting values of the first moments are finally analysed using the NNNLO result for the Bjorken sum rule in order to extract $\alpha_{s}$. The procedure can be justified if the $Q^{2} \operatorname{span}$ of the data around the average value in each experiment is small in comparison to the difference in average $Q^{2}$ between experiments. This is indeed the case for most of the data [3]- [10], with the exception of the SMC data at very large or very small $x$, which however give a relatively small

contribution to the first moments. Although it is generally inconsistent to correct the data 
by using scaling violations at NLO and then to pretend one has a NNNLO accuracy for first moments, in practice this might not be a cause for real concern since the corrections to the data on the truncated first moments from the NLO scaling violations are not large in comparison with the experimental errors and those from the extrapolation in the unmeasured region (see table 2).

We thus first determine for each experiment the value of the first moment of $g_{1}$ in the measured region, evaluated at the average scale $\left\langle Q^{2}\right\rangle$ quoted for that particular experiment, by simply summing the experimental determinations $g_{1}^{e}$ over the bins. Corrected values of $g_{1}$ in order to account for evolution at a common scale are determined according to [8]

$$
\begin{aligned}
& g_{1}\left(x,\left\langle Q^{2}\right\rangle\right) \approx g_{1}^{e}\left(x, Q^{2}\right)+\Delta\left(x, Q^{2},\left\langle Q^{2}\right\rangle\right), \\
& \Delta\left(x, Q^{2},\left\langle Q^{2}\right\rangle\right) \equiv g_{1}^{f}\left(x,\left\langle Q^{2}\right\rangle\right)-g_{1}^{f}\left(x, Q^{2}\right),
\end{aligned}
$$

where $g_{1}^{f}\left(x, Q^{2}\right)$ is a best-fit, which we take from our sets of fits A-D. The fits with $g_{A}$ left as a free parameter must be used here, otherwise the determination of the nonsinglet first moment would be circular. Because all these fits give very similar results in the measured region (compare table 2), we can assume the error in the evolution procedure to be negligible and take the error in this contribution to the moment to be that given by the experimental collaborations. We then complete the moments in the unmeasured region by again using the set of fits A-D, which differ widely in the small $x$ region, but are all consistent both with the experimental data and well understood theoretical principles. Specifically, we take (for each experiment) the average of the difference between the full and truncated integrals for the four fits as an estimate of the extrapolation. The associated uncertainty is then taken to be given by their spread as one varies the functional form of the fit, as well as renormalization and factorization scales, and the value of $\alpha_{s}$ (variations of the thresholds and the value of $a_{8}$ have no significant effect). The measured and extrapolated first moments are then added and the respective errors added in quadrature (see table 4). This is then the best estimate of the experimental determination of the first moments and their associated errors.

In order to determine the isotriplet combinations relevant for the Bjorken sum rule we have repeated the same analysis directly at the level of the isotriplet combinations of first moments, which we can determine separately at $10 \mathrm{GeV}^{2}$ for the SMC experiment, and at $3 \mathrm{GeV}^{2}$ for all of the SLAC experiments (by averaging the neutron data). The error due to the extrapolation is then only sensitive to the uncertainty in the nonsinglet contribution to $g_{1}$ at small $x$ and thus much smaller than the sum in quadrature of the errors in individual first moments: in practice only the variation of the functional form of the fit contributes, because the nonsinglet extrapolation is essentially insensitive to the value of $\alpha_{s}$ and the choice of renormalization and factorization scales. The result, listed in table 4, shows that whereas for the SMC data the dominant uncertainty is in the measured region, for the SLAC data the uncertainty in the extrapolation is comparable to it.

From the isotriplet first moments of table 4 we can then determine $\alpha_{s}\left(m_{Z}\right)$ by fixing $g_{A}$ to 
its value $g_{A}=1.2573 \pm 0.0028$, eq. (13). We find

$$
\alpha_{s}\left(m_{Z}\right)=\left\{\begin{array}{lll}
0.111_{-0.111}^{+0.035} & (\exp ) & \text { SMC only } \\
0.118_{-0.026}^{+0.010} & (\exp ) & \text { SLAC only } \\
0.118_{-0.024}^{+0.010} & (\exp ) & \text { all data }
\end{array}\right.
$$

where for the SMC only case the lower error should be understood as meaning that the uncertainty is $100 \%$ or more. The error in eq. (18) is the purely experimental one due to the error on the isotriplet first moment of table 4 . This error is now so large that it dominates any theoretical errors. For instance, we can estimate a higher twist error by adding to the Bjorken integral a twist four term, with a coefficient estimated from sum rule and renormalon calculations [39]. The value of $\alpha_{s}$ of eq. (18) is then reduced by about 0.004 . We used this as an estimate of the error from higher twist terms in the determination of $\alpha_{s}$ eq. (15) (see table 3).

As a cross-check, we can instead fix $\alpha_{s}$ to its known value $\alpha_{s}\left(m_{Z}\right)=0.118 \pm 0.005$ and extract $g_{A}$ from the sum rule: we then get $g_{A}=1.27 \pm 0.11 \pm 0.05 \pm 0.03$ where the first error is experimental, the second is due to the error on $\alpha_{s}$, and the third is the higher twist error, estimated as above (and used in table 3). These errors are comparable to those found by the direct determination of $g_{A}$ eq. (13), but the experimental error is somewhat larger because only a subset of the information contained in the data is now being used.

We conclude that the result for $\alpha_{s}$ obtained from this procedure is compatible with that from the direct determination eq. (15), but that the error is now considerably larger, in agreement with our previous observation that $\alpha_{s}$ is better constrained by the data in the measured range than by the Bjorken integral alone. The value of $\alpha_{s}\left(m_{Z}\right)$ is very close to that found in ref. 23. but the error is larger because of the error from the small $x$ extrapolation which we no longer assume to follow naive Regge expectations at $\left\langle Q^{2}\right\rangle$. A direct determination of $\alpha_{s}$ from the Bjorken sum rule could however be competitive if data with a wider kinematic coverage in $x$ were available.

\section{Conclusions}

We have performed a global analysis of all data on the polarized structure function $g_{1}$ for proton, deuteron and neutron targets, including data which have only recently become available, using NLO perturbative QCD. We took care to properly estimate the effects of perturbative evolution and the uncertainties in the small $x$ extrapolation when computing first moments. We showed that the data confirm the Bjorken sum rule to within one standard deviation at the $8 \%$ level, and indicate a gluon polarization in the nucleon which is non-zero at the level of two standard deviations. We showed further that the data now provide a reasonably accurate determination of $\alpha_{s}$, consistent with the global average. However we also showed that the Bjorken integral is unfortunately not yet sufficiently well determined to admit a competitive determination of $\alpha_{s}$ using the Bjorken sum rule.

Our determination of $\alpha_{s}$ could be improved by more accurate data on polarization asymmetries in the fixed target region, or by an independent (semi-inclusive) determination of the 
polarized gluon distribution. However the theoretical error from higher order corrections is already the dominant one. The error in our determination of first moments is instead dominated by uncertainties in the small $x$ region, and we suspect that these uncertainties will only be significantly reduced by the measurement of $g_{1}$ at lower $x$ and higher $Q^{2}$, which would be possible at a polarized colliding beam experiment at HERA [45].

Acknowledgement: We thank P. Bosted and A. Deshpande for useful information on the experimental data. 


\section{References}

[1] G. Altarelli, in "The challenging questions", Proc. of the 1989 Erice School, A. Zichichi, ed. (Plenum, New York, 1990);

R.D. Ball, in "The Spin Structure of the Nucleon", Proc. of the 1995 Erice School of Nucleon Structure, B. Frois and V.W. Hughes, ed. hep-ph/9511330;

S. Forte, in the proceedings of the "14th International Conference on Particles and Nuclei (PANIC96)" and "12th International Symposium on High Energy Spin Physics (SPIN96)", hep-ph/9610238;

G. Ridolfi, in the proceedings of the "International Workshop on Deep Inelastic Scattering and Related Phenomena (DIS96)", hep-ph/9610214.

[2] EMC Collaboration, J. Ashman et al., Nucl. Phys. B328 (1989) 1.

[3] SMC Collaboration, D. Adams et al., Phys. Lett. B329 (1994) 399.

[4] E143 Collaboration, K. Abe et al., Phys. Rev. Lett. 74 (1995) 346.

[5] E143 Collaboration, K. Abe et al., Phys. Lett. B364 (1995) 61.

[6] SMC Collaboration, D. Adams et al., Phys. Lett. B357 (1995) 248.

[7] E143 Collaboration, K. Abe et al., Phys. Rev. Lett. 75 (1995) 25.

[8] SMC Collaboration, D. Adams et al., hep-ex/9702005.

[9] E142 Collaboration, P.L. Anthony et al., Phys. Rev. D54 (1996) 6620.

[10] Y.G. Kolomensky for the E154 Collaboration, talk at the "12th International Symposium on High Energy Spin Physics (SPIN96)".

[11] R. Mertig and W. L. van Neerven, Zeit. Phys. C70 (1996) 637;

W. Vogelsang, Phys. Rev. D54 (1996) 2023.

[12] R.D. Ball and A. DeRoeck, in the proceedings of the "International Workshop on Deep Inelastic Scattering and Related Phenomena (DIS96)", hep-ph/9609309, and ref. therein.

[13] J.D. Bjorken, Phys. Rev. 148 (1966) 1467.

[14] J. Ellis and M. Karliner, Phys. Lett. B313 (1993) 131.

[15] G. Altarelli and G. Parisi, Nucl. Phys. B126 (1977) 298.

[16] R.D. Ball, S. Forte and G. Ridolfi, Nucl. Phys. B444 (1995) 287.

[17] R.D. Ball, S. Forte and G. Ridolfi, Phys. Lett. B378 (1996) 255.

[18] F. E. Close and R. G. Roberts, Phys. Lett. B336 (1994) 257.

[19] R. L. Heimann, Nucl. Phys. B64 (1973) 429. 
[20] A. De Rújula et al., Phys. Rev. 10 (1974) 1649.

[21] R.D. Ball and S. Forte, Phys. Lett. B335 (1994) 77; B336 (1994) 77; Acta. Phys. Pol. B26 (1995) 2097.

[22] M. A. Ahmed and G. G. Ross, Phys. Lett. B56 (1975) 385;

M. B. Einhorn and J. Soffer, Nucl. Phys. B74 (1986) 714;

A. Berera, Phys. Lett. B293 (1992) 445.

[23] J. Ellis and M. Karliner, Phys. Lett. B341 (1995) 397; talk in "The Spin Structure of the Nucleon", Proc. of the 1995 Erice School of Nucleon Structure, B. Frois and V.W. Hughes, ed. (hep-ph/9601280).

[24] T. Gehrmann and W. J. Stirling, Phys. Rev. D53 (1996) 6100;

M. Glück et al., Phys. Rev. D53 (1996) 4775.

[25] G. Altarelli and G. G. Ross, Phys. Lett. B212 (1988) 391.

[26] A.V. Efremov and O.V. Teryaev, Dubna preprint E2-88-287 (unpublished).

[27] R.D. Carlitz, J.C. Collins and A.H. Mueller, Phys. Lett. B214 (1988) 229.

[28] G. Altarelli and B. Lampe, Zeit. Phys. C47 (1990) 315.

[29] S. Forte, R.D. Ball and G. Ridolfi, in the proceedings of the "International Workshop on Deep Inelastic Scattering and Related Phenomena (DIS96)", hep-ph/9608399.

[30] L.N. Lipatov, Sov. J. Nucl. Phys. 23 (1977) 338;

E.A. Kuraev, L.N. Lipatov and V.S. Fadin, Sov. Phys. JETP 45 (1977) 199;

Ya. Balitskii and L.N. Lipatov, Sov. J. Nucl. Phys. 28 (1978) 822.

[31] R. Kirschner and L. Lipatov, Nucl. Phys. B213 (1983) 122.

[32] B.I. Ermolaev, S.I. Manaenkov and M.G. Ryskin, Zeit. Phys. C69 (1996) 259;

J. Bartels, B. I. Ermolaev and M. G. Ryskin, Zeit. Phys. C70 (1996) 273; hep-ph/9603204.

[33] NMC Collaboration, M. Arneodo Phys. Lett. B364 (1995) 107.

[34] L. W. Whitlow et al., Phys. Lett. B250 (1990) 193.

[35] L. L. Frankfurt and M. Strikman, Nucl. Phys. A405 (1983) 557;

J. L. Friar et al., Phys. Rev. C42 (1990) 2310;

C. Ciofi degli Atti et al., Phys. Rev. C48 (1993) 968.

[36] S. Bethke, talk at the "High-energy Physics International Euroconference on Quantum Chromodynamics (QCD 96)", hep-ex/9609014;

G. Altarelli, talks at the "NATO Advanced Study Institute on Techniques and Concepts of High-Energy Physics" and at the "Cracow International Symposium on Radiative Corrections (CRAD 96)", hep-ph/9611239;

P.N. Burrows, talk at the "Cracow International Symposium on Radiative Corrections (CRAD 96)", hep-ph/9612007. 
[37] F. E. Close and R. G. Roberts, Phys. Lett. B316 (1993) 165.

[38] B. Ehrnsperger and A. Schäfer, Phys. Lett. B348 (1995) 619;

J. Lichtenstadt and H. J. Lipkin, Phys. Lett. B353 (1995) 119;

J. Dai et al., Phys. Rev. D53 (1996) 273;

P. G. Ratcliffe, Phys. Lett. B365 (1996) 383.

[39] See L. Mankiewicz, E. Stein and A. Schäfer, hep-ph/9510418 and ref. therein.

[40] Particle Data Group, Phys. Rev. D54 (1996) 1.

[41] G. Altarelli and G. Ridolfi, Nucl. Phys. B (Proc. Suppl.) 39B (1995) 106.

[42] J. Ellis and R. L. Jaffe, Phys. Rev. D9 (1974) 1444.

[43] S. Forte, Phys. Lett. B224 (1989) 189; Nucl. Phys. B331 (1990) 1;

G.M. Shore and G. Veneziano, Phys. Lett. B244 (1990) 75; Nucl. Phys. B381 (1992) 23;

R.D. Ball, Phys. Lett. B266 (1991) 473.

[44] S. G. Gorishny and S. A. Larin, Phys. Lett. B172 (1986) 109;

S. A. Larin and J. A. M. Vermaseren, Phys. Lett. B259 (1991) 345.

[45] R.D. Ball et al, in the proceedings of the 'Workshop on Future Physics at HERA', Hamburg, hep-ph/9609515. 


\begin{tabular}{|l|l|l|l|l|}
\hline Parameters & $\mathrm{A}$ & $\mathrm{B}$ & $\mathrm{C}$ & $\mathrm{D}$ \\
\hline d.o.f. & $114-11$ & $114-11$ & $114-8$ & $114-8$ \\
$Q_{0}^{2} / \mathrm{GeV}^{2}$ & 1 & 1 & 0.3 & 0.3 \\
\hline$\eta_{\Sigma}$ & $0.408 \pm 0.041$ & $0.410 \pm 0.039$ & $0.422 \pm 0.026$ & $0.492 \pm 0.036$ \\
$\alpha_{\Sigma}$ & $0.741 \pm 0.353$ & $1.710 \pm 0.416$ & $2.600 \pm 0.964$ & $0.5 \quad$ (fixed) \\
$\beta_{\Sigma}$ & $3.105 \pm 1.049$ & $2.735 \pm 0.466$ & $3.359 \pm 1.210$ & $1.039 \pm 0.241$ \\
$\gamma_{\Sigma}$ & $0.185 \pm 2.496$ & $0 \quad$ (fixed) & $0 \quad$ (fixed) & $0 \quad$ (fixed) \\
$\eta_{g}$ & $1.068 \pm 0.403$ & $1.032 \pm 0.330$ & $0.479 \pm 0.095$ & $0.650 \pm 0.104$ \\
$\alpha_{g}$ & $-0.597 \pm 0.286$ & $2.970 \pm 0.611$ & $0.217 \pm 0.319$ & $0.5 \quad$ (fixed) \\
$\beta_{g}$ & $0.831 \pm 2.322$ & $1.286 \pm 0.895$ & $15 \quad$ (fixed) & $13.19 \pm 9.57$ \\
$\gamma_{g}$ & $0.185 \pm 2.496$ & $21.2 \pm 22.5$ & $0 \quad$ (fixed) & $-0.548 \pm 9.139$ \\
$g_{A}$ & $1.168 \pm 0.052$ & $1.234 \pm 0.066$ & $1.146 \pm 0.038$ & $1.141 \pm 0.036$ \\
$a_{8}$ & $0.579($ fixed) & $0.579 \quad($ fixed) & $0.579 \quad($ fixed) & 0.579 (fixed) \\
$\alpha_{N S}$ & $-0.537 \pm 0.057$ & $1.656 \pm 0.166$ & $0.765 \pm 0.228$ & $0.5 \quad$ (fixed) \\
$\beta_{N S}$ & $2.503 \pm 0.274$ & $5.320 \pm 0.251$ & $2.087 \pm 0.448$ & $2.622 \pm 0.410$ \\
$\gamma_{N S}$ & $17.46 \pm 8.43$ & $-0.229 \pm 0.105$ & $0 \quad$ (fixed) & $5.024 \pm 4.647$ \\
\hline$\chi^{2}$ & 83.7 & 83.0 & 83.8 & 90.9 \\
$\chi^{2} /$ d.o.f. & 0.813 & 0.806 & 0.790 & 0.858 \\
\hline$\Delta g\left(1,1 \mathrm{GeV}^{2}\right)$ & $1.07 \pm 0.40$ & $1.03 \pm 0.33$ & $1.61 \pm 0.32$ & $2.08 \pm 0.34$ \\
$a_{0}\left(10 \mathrm{GeV}^{2}\right)$ & $0.15 \pm 0.07$ & $0.16 \pm 0.05$ & $0.05 \pm 0.04$ & $0.02 \pm 0.04$ \\
\hline
\end{tabular}

Table 1: Results of fits A-D described in the text

\begin{tabular}{|l|l|l|l|l|l|l|}
\hline & SMC $: \mathrm{p}$ & $\mathrm{E} 143: \mathrm{p}$ & SMC $: \mathrm{d}$ & $\mathrm{E} 143: \mathrm{d}$ & $\mathrm{E} 142: \mathrm{n}$ & $\mathrm{E} 154: \mathrm{n}$ \\
\hline$\left\langle Q^{2}\right\rangle / \mathrm{GeV}^{2}$ & 10 & 3 & 10 & 3 & 2 & 5 \\
\hline Meas. Range: Exp. & 0.1310 & 0.1200 & 0.0379 & 0.0400 & -0.0280 & -0.0370 \\
Exp. Error & \pm 0.0156 & \pm 0.0089 & \pm 0.0079 & \pm 0.0050 & \pm 0.0085 & \pm 0.0108 \\
\hline$A_{1}$ ind. $Q^{2}$ & 0.1350 & 0.1059 & 0.0442 & 0.0398 & -0.0293 & -0.0364 \\
\hline Meas. Range: A & 0.1256 & 0.1070 & 0.0430 & 0.0378 & -0.0328 & -0.0338 \\
Meas. Range: B & 0.1268 & 0.1061 & 0.0425 & 0.0373 & -0.0326 & -0.0342 \\
Meas. Range: C & 0.1280 & 0.1084 & 0.0462 & 0.0401 & -0.0289 & -0.0316 \\
Meas. Range: D & 0.1308 & 0.1083 & 0.0492 & 0.0402 & -0.0306 & -0.0308 \\
\hline Full Range: A & 0.1171 & 0.1137 & 0.0275 & 0.0265 & -0.0600 & -0.0614 \\
Full Range: B & 0.1232 & 0.1197 & 0.0286 & 0.0276 & -0.0637 & -0.0654 \\
Full Range: C & 0.1039 & 0.0999 & 0.0160 & 0.0144 & -0.0707 & -0.0716 \\
Full Range: D & 0.0990 & 0.0946 & 0.0115 & 0.0094 & -0.0754 & -0.0759 \\
\hline
\end{tabular}

Table 2: Determination of the first moment $\Gamma_{1}\left(\left\langle Q^{2}\right\rangle\right)$ eq. (四). For each experiment we display the average value of $Q^{2}$ and the contribution to the first moments from the measured range of $x$, as given, first, by the experimental collaborations, with the corresponding total (statistical and systematic) error, then by summing over experimental bins while evolving the data assuming scale independent asymmetries, and finally as obtained from integration of the fits A-D. In the last four rows the complete first moments obtained from the fits A-D are shown. 


\begin{tabular}{|l|l|l|l|l|l|}
\hline & $g_{A}$ & $\Delta \Sigma$ & $\Delta g$ & $a_{0}$ & $\alpha_{s}$ \\
\hline experimental & \pm 0.05 & \pm 0.04 & \pm 0.4 & \pm 0.05 & ${ }_{-0.005}^{+0.004}$ \\
\hline fitting & \pm 0.05 & \pm 0.05 & \pm 0.5 & \pm 0.07 & \pm 0.001 \\
$\alpha_{s} \& a_{8}$ & \pm 0.03 & \pm 0.01 & \pm 0.2 & \pm 0.02 & \pm 0.000 \\
thresholds & \pm 0.02 & \pm 0.05 & \pm 0.1 & \pm 0.01 & \pm 0.003 \\
higher orders & \pm 0.03 & \pm 0.04 & \pm 0.6 & ${ }_{-0.07}^{+0.15}$ & ${ }_{-0.004}^{+0.007}$ \\
higher twists & \pm 0.03 & - & - & - & \pm 0.004 \\
\hline theoretical & \pm 0.07 & \pm 0.08 & \pm 0.8 & ${ }_{-0.010}^{+0.17}$ & ${ }_{-0.006}^{+0.009}$ \\
\hline
\end{tabular}

Table 3: Contributions to the errors in the determination of the quantities $g_{A}, \Delta \Sigma(1)$, $\Delta g\left(1,1 \mathrm{GeV}^{2}\right), a_{0}(\infty)$ and $\alpha_{s}\left(m_{Z}\right)$ from the fits described in the text.

\begin{tabular}{|l|l|l|l|}
\hline & $\left\langle Q^{2}\right\rangle$ & Meas. Range & Full Range \\
\hline SMC : p & 10 & $0.133 \pm 0.016$ & $0.116 \pm 0.022$ \\
SMC : d & 10 & $0.045 \pm 0.008$ & $0.021 \pm 0.016$ \\
SMC : I=1 & 10 & $0.176 \pm 0.036$ & $0.191 \pm 0.037$ \\
\hline E143 : p & 3 & $0.108 \pm 0.009$ & $0.107 \pm 0.017$ \\
E143 : d & 3 & $0.040 \pm 0.005$ & $0.021 \pm 0.014$ \\
E142 : n & 2 & $-0.032 \pm 0.009$ & $-0.068 \pm 0.015$ \\
E154 : n & 5 & $-0.034 \pm 0.011$ & $-0.070 \pm 0.015$ \\
SLAC $: \mathrm{I}=1$ & 3 & $0.139 \pm 0.013$ & $0.177 \pm 0.018$ \\
\hline
\end{tabular}

Table 4: Best estimates and errors for the truncated and the complete first moments for the target and the average $Q^{2}$ that correspond to each experiment. The isotriplet first moments for the SMC experiment, and for all the SLAC experiments combined are also given. 

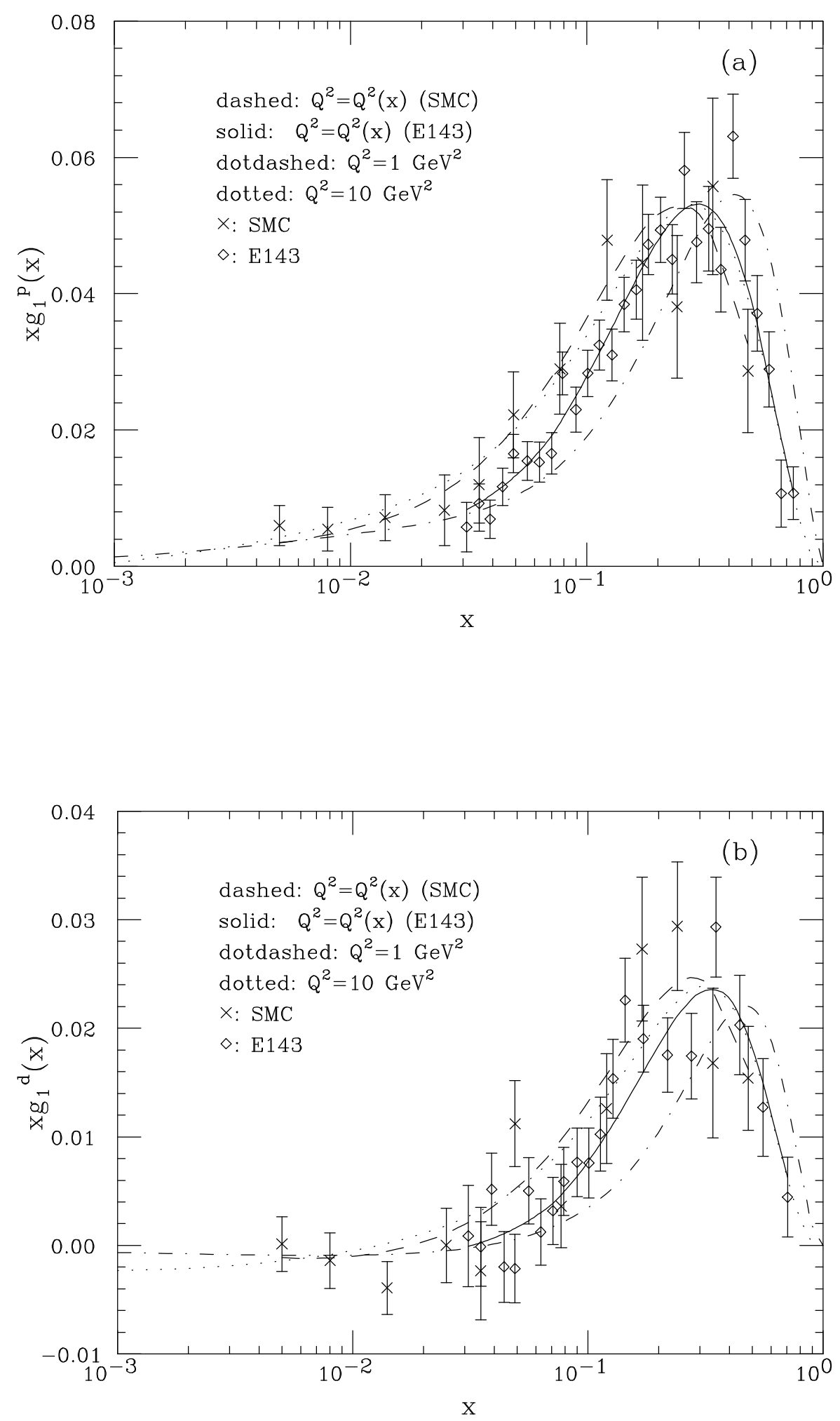


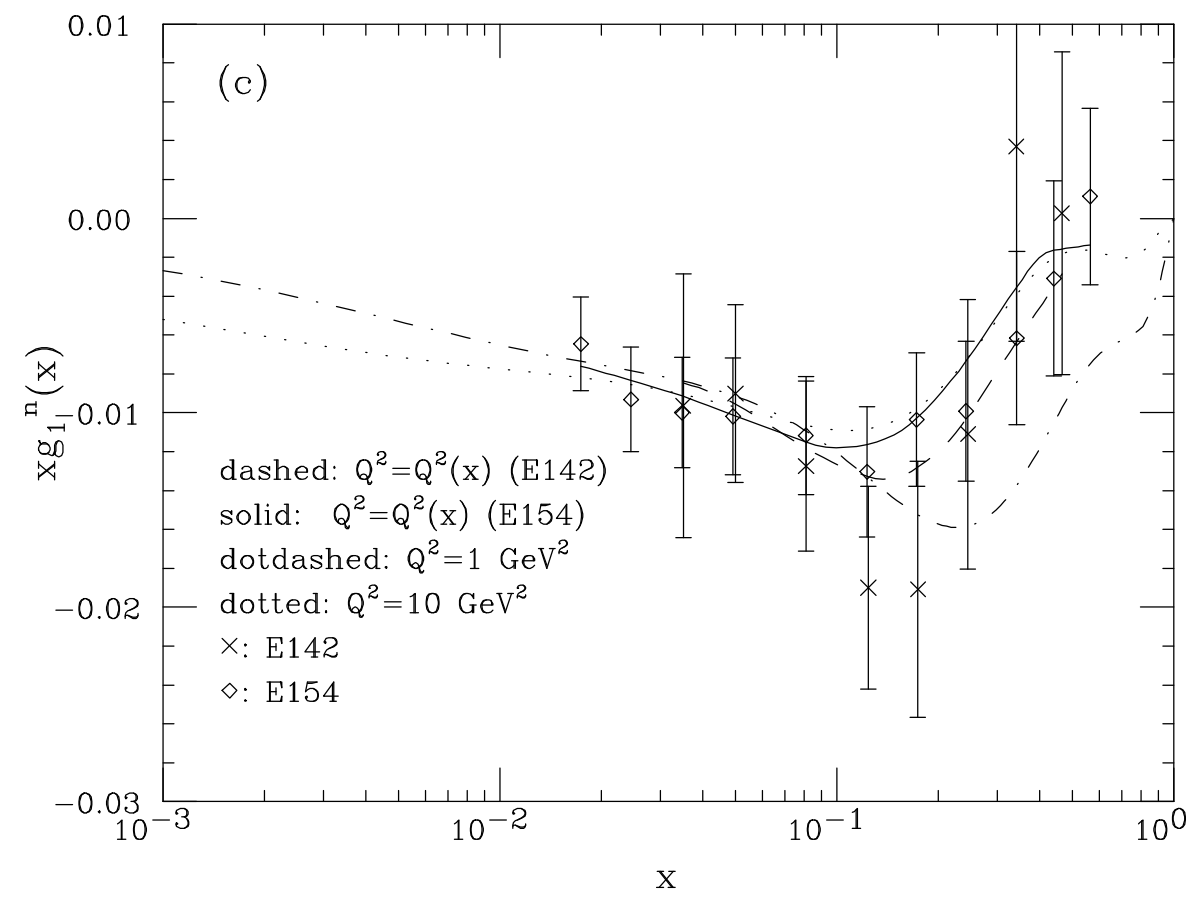

Figure 1: Plots of $x g_{1}\left(x, Q^{2}\right)$ for fit $B$ for (a) proton (b) deuterium (c) and neutron targets. The curves correspond to $Q^{2}=1 \mathrm{GeV}^{2}$ (dotdashed), $Q^{2}=10 \mathrm{GeV}^{2}$ (dotted), and $Q^{2}(x)$ of the various experiments: E143 (solid) and SMC (dashed) for figs. $a-b$ and E142 (solid) and E145 (dashed) for fig. c. The data points with total errors are also shown. 

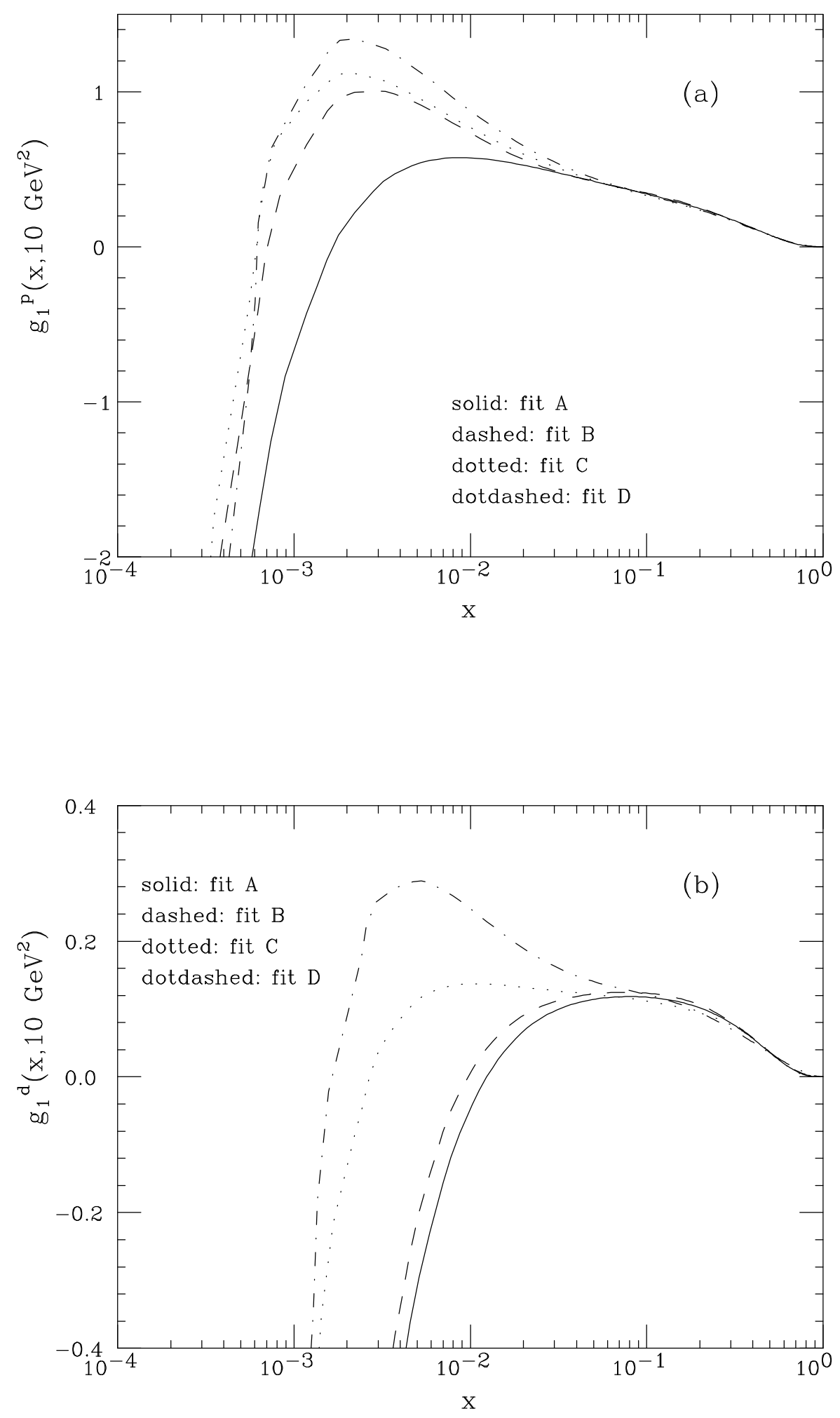


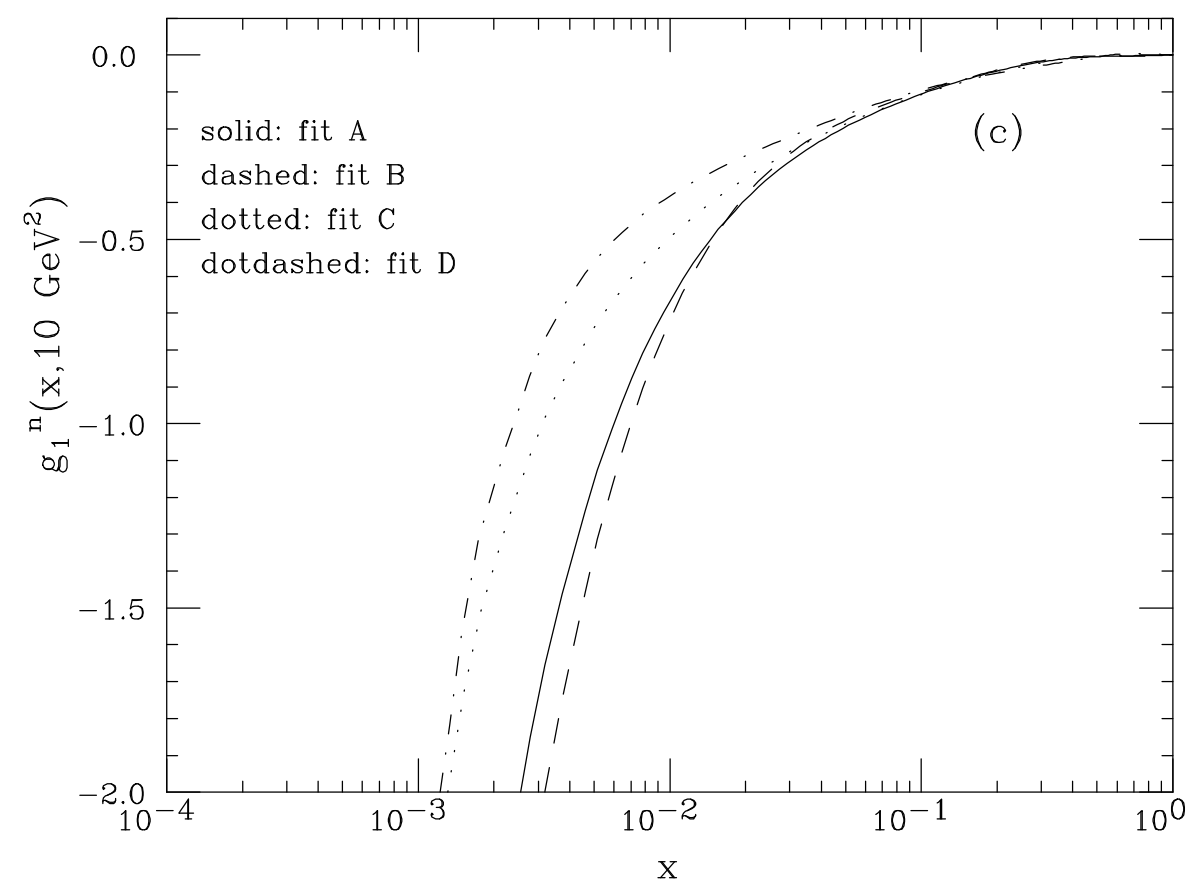

Figure 2: Plots of $g_{1}\left(x, Q^{2}\right)$ for (a) proton (b) deuterium (c) and neutron targets for fits $A-D$ at $Q^{2}=10 \mathrm{GeV}^{2}$.

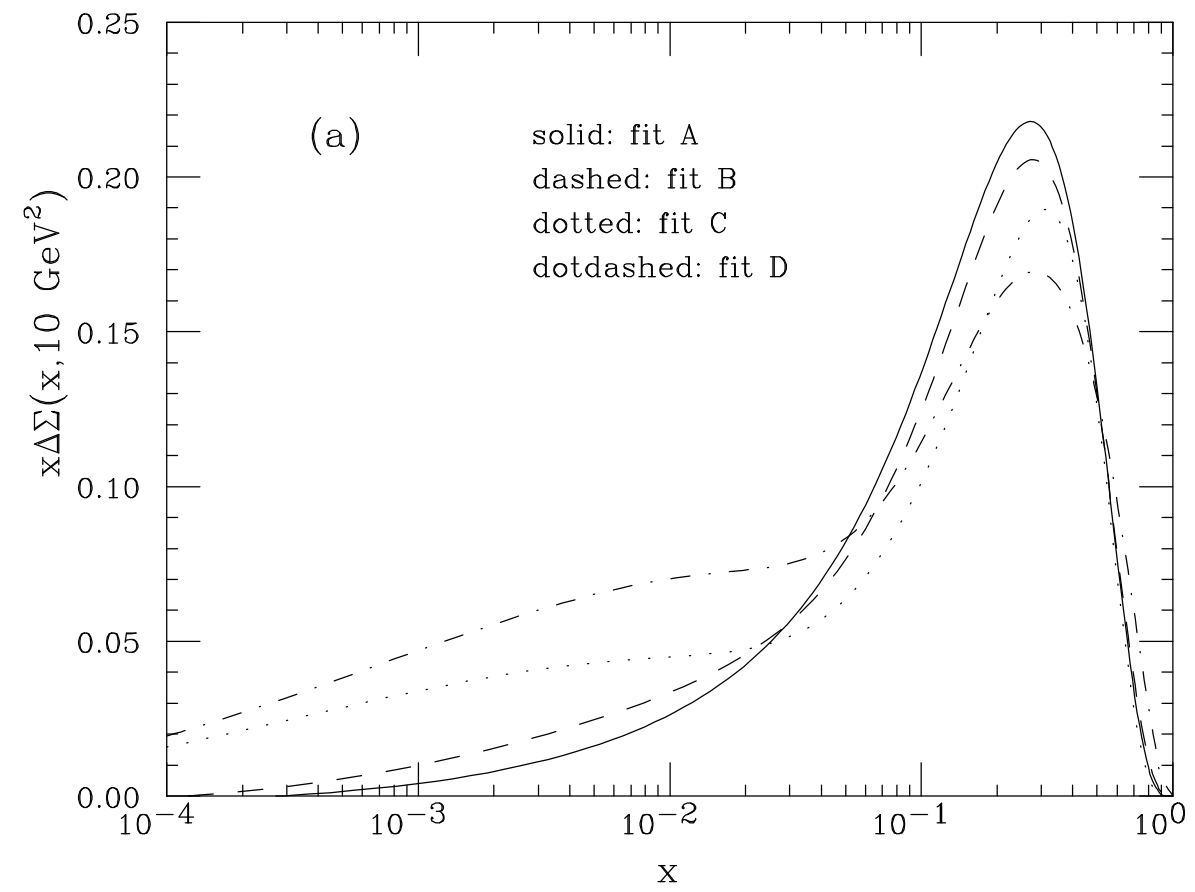



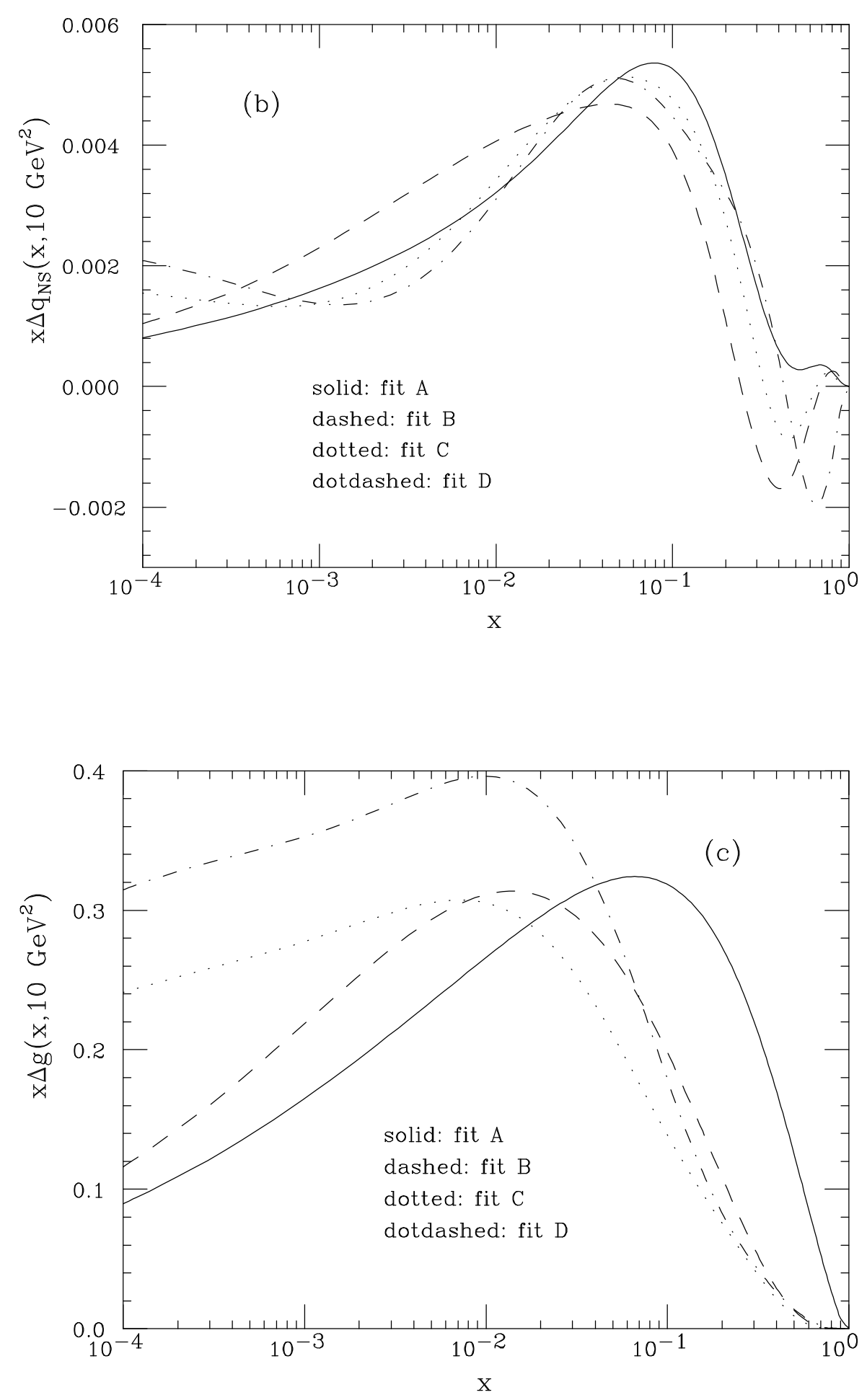

Figure 3: Polarized quark singlet (a) nonsinglet (b) and gluon (c) distributions for fits $A-D$ at $Q^{2}=10 \mathrm{GeV}^{2}$. 